\title{
GENERALIZATIONS OF CAUCHY'S SUMMATION THEOREM FOR SCHUR FUNCTIONS
}

\author{
G. E. ANDREWS, I. P. GOULDEN AND D. M. JACKSON
}

\begin{abstract}
Cauchy's summation theorem for Schur functions is generalized, and a number of related results are given. The result is applied to a combinatorial problem involving products of pairs of permuations, by appeal to properties of the group algebra of the symmetric group.
\end{abstract}

1. Introduction. Although interest in the algebra of symmetric functions was originally prompted by work in representation theory, this algebra, the group algebra of the symmetric group and the character theory of the symmetric group play a significant part in combinatorial theory (see, for example, Bannai and Ito [1], Macdonald [6]), and related areas (Diaconis [2]).

In this paper we prove a summation theorem (Theorem 2.1) which gives an explicit evaluation of

$$
\sum_{\theta} \prod_{i=1}^{n}\left(x-\theta_{i}-n+i\right) s_{\theta}\left(y_{1}, \ldots, y_{n}\right) s_{\theta}\left(w_{1}, \ldots, w_{m}\right),
$$

where the sum is over all partitions $\theta$, and $s_{\theta}$ is a Schur function. This is of interest since it is a generalization of the classical result of Cauchy (see [6, p. 33] for a proof):

$$
\sum_{\theta} s_{\theta}\left(y_{1}, \ldots, y_{n}\right) s_{\theta}\left(w_{1}, \ldots, w_{m}\right)=\prod_{j=1}^{n} \prod_{k=1}^{m}\left(1-y_{j} w_{k}\right)^{-1}
$$

In $\S 3$, this summation theorem is used to derive a generalization of Gessel's [3] result on counting 3-rowed Latin rectangles with respect to the number of cycles in each row, considered as permuations. We also derive (Proposition 3.2) an explicit expression for a useful character sum associated with the symmetric group.

$\S 4$ contains a number of results for symmetric functions that are equivalent to Theorem 2.1. These include a generalization of Cauchy's summation theorem for

$$
\sum_{\theta} s_{\theta}\left(y_{1}, \ldots, y_{n}\right) s_{\tilde{\theta}}\left(w_{1}, \ldots, w_{m}\right)
$$

and a summation theorem for

$$
\sum_{\theta} s_{\theta}\left(y_{1}, \ldots, y_{n}\right) S_{\theta}\left(w_{1}, \ldots, w_{m} ; z\right)
$$

where $S_{\theta}$ is a symmetric function related to Hall-Littlewood polynomials.

Received by the editors October 8, 1987 .

1980 Mathematics Subject Classification (1985 Revision). Primary 05A15, 05A19; Secondary $15 \mathrm{~A} 15$. 
In $\S 5$ we give a number of related determinant evaluations. Among the latter is a result due to Borchardt $[\mathbf{1 0}]$ relating the permanent and the determinant of the Cauchy matrix (whose $i j$-element is $1 /\left(1-y_{i} w_{j}\right)$ ).

We have relied heavily on properties of the algebra of symmetric functions. In the interest of brevity, we have not stated these, but have cited the appropriate pages of Macdonald [6], and our notation accords with his.

The following notation is needed. A partition $\theta=\left(\theta_{1}, \theta_{2}, \ldots\right)$ is a nonincreasing, possibly infinite, sequence of nonnegative integers called parts. The number of positive parts is denoted by $l(\theta)$, the sum of the parts is denoted by $|\theta|$ and the number parts of size one is denoted by $g(\theta)$. We say that $\theta$ is a partition of $|\theta|$ and we write $\theta \vdash N$. The conjugate of $\theta$ is denoted by $\tilde{\theta}$. The partition $(n-1, n-$ $2, \ldots, 1,0, \ldots)$ is denoted by $\delta$.

Throughout this paper $e_{k}\left(y_{1}, \ldots, y_{n}\right), h_{k}\left(y_{1}, \ldots, y_{n}\right), p_{k}\left(y_{1}, \ldots, y_{n}\right)$, and $s_{\theta}\left(y_{1}, \ldots, y_{n}\right)$, denote the elementary, complete, power sum and Schur symmetric functions, respectively, on the (commuting) indeterminates $y_{1}, \ldots, y_{n}$. When $n=\infty$, these will be abbreviated to $e_{k}(y), h_{k}(y), p_{k}(y)$ and $s_{\theta}(y)$, respectively, and, when no confusion arises, to $e_{k}, h_{k}, p_{k}$ and $s_{\theta}$.

There is a natural bijection between the partitions of weight $N$ and the set of conjugacy classes of the symmetric group, $\mathbf{S}_{N}$, on $N$ symbols in which the number of parts of a partition is the number of (disjoint) cycles in an arbitrary element of the corresponding conjugacy class. Thus $l(\alpha)$ is also the number of cycles in an arbitrary element $\alpha$ of the conjugacy class, $C_{\theta}$, corresponding to $\theta$. Similarly, $g(\alpha)$ is the number of 1-cycles (fixed points) in an arbitrary element $\alpha$ of $C_{\theta}$. The size of $C_{\theta}$ is denoted by $h^{\theta}$. Let $\chi_{\theta}$ be the irreducible (ordinary) character associated with $C_{\theta}$ and let $f^{\theta}$ be the degree of $\chi_{\theta}$. The value at any element of $C_{\alpha}$ is denoted by $\chi_{\alpha}^{\theta}$. The signum of a permutation $\pi$ is denoted by $\operatorname{sgn}(\pi)$.

Let $\mathbf{R}$ be any commutative ring. Let $[\cdot]_{\mathbf{R}}$ be the usual coefficient operator on the ring $\mathbf{R}[[x]]$ of formal power series in the indeterminate $x$ with $\mathbf{R}$ as coefficient ring. Clearly, this operator acts linearly on the ring. In general, the ring will be understood from the context, so the subscript of the coefficient operator will be suppressed. Similarly, to avoid unnecessary detail, we have defined mappings by their elementwise action, since their domain and range are clear from the context. Unless otherwise stated, the operand of a mapping defined on a ring is the whole of the expression to its right.

Throughout, $(x)_{k}=x(x-1) \cdots(x-k+1)$ and $(x)^{(k)}=x(x+1) \cdots(x+k-1)$, the falling and rising factorials, respectively. If the $i j$-element of a matrix $M$ is $m_{i j}$, for $i \in I, j \in J$, then the determinant of $M$ is denoted by

$$
\left\|\left[m_{i j}\right]_{\substack{i \in I \\ j \in J}}\right\|
$$

When $I=J=\{1, \ldots, k\}$, this is simplified to $\left\|m_{i j}\right\|_{k \times k}$. We denote $\left\|y_{j}^{\theta_{i}}\right\|_{n \times n}$ by $a_{\theta}\left(y_{1}, \ldots, y_{n}\right)$, or by $a_{\theta}$, when no confusion arises.

2. A summation theorem for Schur functions. In this section we derive the main result of this paper, by showing that it is equivalent to a polynomial identity. This is proved by induction on $n+m$. 
THEOREM 2.1. For nonnegative integers $n$ and $m$,

$$
\begin{aligned}
& \sum_{\theta} \prod_{i=1}^{n}\left(x-\theta_{i}-n+i\right) s_{\theta}\left(y_{1}, \ldots, y_{n}\right) s_{\theta}\left(w_{1}, \ldots, w_{m}\right) \\
& \quad=\prod_{j=1}^{n} \prod_{k=1}^{m}\left(1-y_{j} w_{k}\right)^{-1}\left[t_{1} \cdots t_{n}\right]\left(1+t_{1}+\cdots+t_{n}\right)^{x} \prod_{k=1}^{m}\left(1-\sum_{j=1}^{n} \frac{t_{j} y_{j} w_{k}}{1-y_{j} w_{k}}\right) .
\end{aligned}
$$

ProOF. First we rewrite the left-hand side by $[6$, p. 24] as

$$
\sum_{\substack{\theta \\ l(\theta) \leq n}} \prod_{i=1}^{n}\left(x-\theta_{i}-n+i\right) \frac{a_{\delta+\theta}}{a_{\delta}} s_{\theta}\left(w_{1}, \ldots, w_{m}\right) .
$$

Let $Y_{z}$ denote the formal partial differential operator $\prod_{i=1}^{n}\left(x-\left(y_{i}-z\right) \partial / \partial y_{i}\right)$. Now

$$
\prod_{i=1}^{n}\left(x-\theta_{i}-n+i\right) a_{\delta+\theta}=\sum_{\sigma \in S_{n}} \operatorname{sgn}(\sigma) \prod_{i=1}^{n}\left(x-\theta_{i}-n+i\right) y_{\sigma(i)}^{\theta_{i}+n-i}=Y_{0} a_{\delta+\theta}
$$

so the left-hand side becomes

$$
a_{\delta}^{-1} Y_{0} \sum_{\substack{\theta \\ l(\theta) \leq n}} a_{\delta+\theta} s_{\theta}\left(w_{1}, \ldots, w_{m}\right)=a_{\delta}^{-1} Y_{0} a_{\delta} \prod_{j=1}^{n} \prod_{k=1}^{m}\left(1-y_{j} w_{k}\right)^{-1}
$$

from Cauchy's summation theorem. Now multiply through by $a_{\delta}$, which gives the following equivalent formulation of the result:

$$
\begin{array}{r}
Y_{0} a_{\delta} \prod_{j=1}^{n} \prod_{k=1}^{m}\left(1-y_{j} w_{k}\right)^{-1}=a_{\delta} \prod_{j=1}^{n} \prod_{k=1}^{m}\left(1-y_{j} w_{k}\right)^{-1}\left[t_{1} \cdots t_{n}\right] \\
\cdot\left(1+t_{1}+\cdots+t_{n}\right)^{x} \prod_{k=1}^{m}\left(1-\sum_{j=1}^{n} \frac{t_{j} y_{j} w_{k}}{1-y_{j} w_{k}}\right) .
\end{array}
$$

Now replace each $w_{k}$ by $w_{k}^{-1}$, and then each $y_{j}$ by $y_{j}-z$ and each $w_{k}$ by $w_{k}-z$; next multiply on both sides by $\prod_{j=1}^{n} \prod_{k=1}^{m}\left(w_{k}-z\right)^{-1}$. As a result we have the following equivalent formulation of (1):

$$
\begin{aligned}
Y_{z} a_{\delta} \prod_{j=1}^{n} \prod_{k=1}^{m}\left(w_{k}-y_{j}\right)^{-1}=a_{\delta} \prod_{j=1}^{n} \prod_{k=1}^{m}\left(w_{k}-y_{j}\right)^{-1}\left[t_{1} \cdots t_{n}\right] \\
\cdot\left(1+t_{1}+\cdots+t_{n}\right)^{x} \prod_{k=1}^{m}\left(1+\sum_{j=1}^{n} \frac{t_{j}\left(z-y_{j}\right)}{w_{k}-y_{j}}\right) .
\end{aligned}
$$

We first prove (2) in the special cases $n=0$ and $m=0$.

Case (i): $n=0$. Both sides are equal to 1 , so (2) is true in this case.

Case (ii): $m=0$. The right-hand side is

$$
a_{\delta}\left[t_{1} \cdots t_{n}\right]\left(1+t_{1}+\cdots+t_{n}\right)^{x}=a_{\delta}\left(\begin{array}{l}
x \\
n
\end{array}\right) n !=a_{\delta}(x)_{n} .
$$


The left-hand side is

$$
\begin{aligned}
Y_{z} a_{\delta} & =Y_{z}\left\|y_{i}^{n-j}\right\|_{n \times n}=\left\|\left(x-\left(y_{i}-z\right) \frac{\partial}{\partial y_{i}}\right) y_{i}^{n-j}\right\|_{n \times n} \\
& =\left\|(x-n+j) y_{i}^{n-j}+z(n-j) y_{i}^{n-j-1}\right\|_{n \times n} \\
& =\left\|(x-n+j) y_{i}^{n-j}\right\|_{n \times n}=(x)_{n}\left\|y_{i}^{n-j}\right\|_{n \times n}=(x)_{n} a_{\delta}
\end{aligned}
$$

and (2) is true in this case.

We prove (2) by induction on $n+m$ where $n \geq 0, m \geq 0$. The case $n+m=0$ is accounted for in Cases (i) and (ii) above. The induction hypothesis is that the result is true for all cases $n+m<N, n \geq 0, m \geq 0$. We must now prove the result is true for $n+m=N, n \geq 1, m \geq 1$. (The cases $n=0$ and $m=0$ are already accounted for.)

The left- and right-hand sides are polynomials in $z$ of degree at most $n$ and $m$, respectively, in $z$, so it is sufficient to prove that they agree on $n+m$ values of $z$, since $n+m>n$ and $n+m>m$ for $n \geq 1, m \geq 1$, so these polynomials agree on more values than either of their degrees.

First we consider $z=y_{n}$ (by a permutation of the indices, this argument works for all of $\left.z=y_{1}, \ldots, y_{n}\right)$. Substituting $y_{n}$ for $z$ in (2), then replacing $y_{n}$ by $\xi$ to draw attention to it, and multiplying both sides by $\prod_{k=1}^{m}\left(w_{k}-\xi\right)$, gives

$$
\begin{aligned}
& x \prod_{i=1}^{n-1}\left(x-\left(y_{i}-\xi\right) \frac{\partial}{\partial y_{i}}\right)\left(y_{1}-\xi\right) \cdots\left(y_{n-1}-\xi\right) \frac{a_{\delta}\left(y_{1}, \ldots, y_{n-1}\right)}{\prod_{j=1}^{n-1} \prod_{k=1}^{m}\left(w_{k}-y_{j}\right)} \\
& =\left(y_{1}-\xi\right) \cdots\left(y_{n-1}-\xi\right) \frac{a_{\delta}\left(y_{1}, \ldots, y_{n-1}\right)}{\prod_{j=1}^{n-1} \prod_{k=1}^{m}\left(w_{k}-y_{j}\right)} \\
& \cdot\left[t_{1} \cdots t_{n}\right]\left(1+t_{1}+\cdots+t_{n}\right)^{x} \prod_{k=1}^{m}\left(1+\sum_{j=1}^{n-1} \frac{t_{j}\left(\xi-y_{j}\right)}{w_{k}-y_{j}}\right) .
\end{aligned}
$$

But, for any formal power series $F$ in $y_{1}, \ldots, y_{n-1}$, and $i=1, \ldots, n-1$,

$$
\begin{aligned}
\left(x-\left(y_{i}-\xi\right) \frac{\partial}{\partial y_{i}}\right)\left(y_{i}-\xi\right) F & =x\left(y_{i}-\xi\right) F-\left(y_{i}-\xi\right)\left\{F+\left(y_{i}-\xi\right) \frac{\partial F}{\partial y_{i}}\right\} \\
& =\left(y_{i}-\xi\right)\left(x-1-\left(y_{i}-\xi\right) \frac{\partial}{\partial y_{i}}\right) F .
\end{aligned}
$$

Also,

$$
\begin{aligned}
& {\left[t_{1} \cdots t_{n}\right]\left(1+t_{1}+\cdots+t_{n}\right)^{x} \prod_{k=1}^{m}\left(1+\sum_{j=1}^{n-1} \frac{t_{j}\left(\xi-y_{j}\right)}{w_{k}-y_{j}}\right)} \\
& =x\left[t_{1} \cdots t_{n-1}\right]\left(1+t_{1}+\cdots+t_{n-1}\right)^{x-1} \prod_{k=1}^{m}\left(1+\sum_{k=1}^{n-1} \frac{t_{j}\left(\xi-y_{j}\right)}{w_{k}-y_{j}}\right) .
\end{aligned}
$$

If we apply (4) to the left-hand side of (3), and (5) to the right-hand side of (3), and then divide both sides by $x \prod_{j=1}^{n-1}\left(y_{j}-\xi\right)$, we obtain (2) with $n$ replaced by 
$n-1$ and $x$ replaced by $x-1$, which we have assumed to be true by the induction hypothesis, so (3) is true.

Now we consider $z=w_{m}$ (by a permutation of the indices this argument works for all of $\left.z=w_{1}, \ldots, w_{m}\right)$. Substituting $w_{m}$ for $z$ in (2), then replacing $w_{m}$ by $\alpha$ to draw attention to it, gives

$$
\begin{aligned}
& Y_{\alpha} \frac{a_{\delta}}{\left(\alpha-y_{1}\right) \cdots\left(\alpha-y_{n}\right)} \prod_{j=1}^{n} \prod_{k=1}^{m-1}\left(w_{k}-y_{j}\right)^{-1} \\
& =\frac{a_{\delta}}{\left(\alpha-y_{1}\right) \cdots\left(\alpha-y_{n}\right)} \prod_{j=1}^{n} \prod_{k=1}^{m-1}\left(w_{k}-y_{j}\right)^{-1} \\
& \quad \cdot\left[t_{1} \cdots t_{n}\right]\left(1+t_{1}+\cdots+t_{n-1}\right)^{x+1} \prod_{k=1}^{m-1}\left(1+\sum_{j=1}^{n} \frac{t_{j}\left(\alpha-y_{j}\right)}{w_{k}-y_{j}}\right) .
\end{aligned}
$$

But, for any formal power series $G$ in $y_{1}, \ldots, y_{n}$, and $i=1, \ldots, n$,

$$
\left(x-\left(y_{i}-\alpha\right) \frac{\partial}{\partial y_{i}}\right) \frac{G}{\alpha-y_{i}}=\frac{1}{\alpha-y_{i}}\left(x+1-\left(y_{i}-\alpha\right) \frac{\partial}{\partial y_{i}}\right) G .
$$

Now, apply this result to the left-hand side of (6), and multiply both sides by $\prod_{j=1}^{n}\left(\alpha-y_{j}\right)$. This gives (2) with $m$ replaced by $m-1$ and $x$ replaced by $x+1$, which is true by the induction hypothesis, so (6) is true.

Thus (2) is true by induction on $n+m$ for all nonnegative integers $n$ and $m$, and so is the given result, which is equivalent to (2).

Cauchy's summation theorem is obtained by applying $\left[x^{n}\right]$ to both sides of Theorem 2.1.

Sagan [8] has pointed out that extracting the coefficient of $(x)_{n-k}$ from both sides of Theorem 2.1 yields the equivalent statement

$$
\begin{aligned}
\sum_{\theta} & \sum_{1 \leq i_{1}<\cdots i_{k-1}<n}\left(\theta_{i_{1}}+k-1\right)\left(\theta_{i_{2}}+k-2\right) \cdots\left(\theta_{i_{k-1}}+1\right) \\
& \cdot\left(\theta_{i_{k-1}+1}+\cdots+\theta_{n}\right) s_{\theta}\left(y_{1}, \ldots, y_{n}\right) s_{\theta}\left(w_{1}, \ldots, w_{m}\right) \\
= & \prod_{i=1}^{n} \prod_{j=1}^{m} \frac{1}{1-y_{i} w_{j}} \sum_{\substack{j_{1}<\cdots<j_{k} \\
i_{1} \neq \cdots \neq i_{k}}} \prod_{l=1}^{k} \frac{y_{j_{l}} w_{i_{l}}}{1-y_{j_{l}} w_{i_{l}}} .
\end{aligned}
$$

Can one obtain a bijective proof of Theorem 2.1 by applying a refinement of the Robinson-Schensted algorithm [9] to a combinatorial interpretation of this in terms of pairs of matrices and plane partitions?

3. A combinatorial application. Consider the generating functions for pairs of permutations defined by

$$
L(t, u, v, p, q, r)=\sum_{N \geq 0} \frac{t^{N}}{N !} \sum_{(a, b) \in \mathbf{S}_{N}^{2}} u^{l(a)} v^{l(b)} p^{g(a b)} q^{g(a)} r^{g(b)} .
$$

Thus, for example

$$
L(t, u, v, 1,1,1)=\sum_{N \geq 0} \frac{t^{N}}{N !}(u)^{(N)}(v)^{(N)}
$$


since, from [6, p. 22]

$$
(u)^{(N)}=\sum_{a \in \mathbf{S}_{N}} u^{l(a)}
$$

A three rowed reduced Latin rectangle on $N$ columns is a $3 \times N$ array in which the first row contains the identity permutation on $N$ symbols, and the other rows contain permutations on $N$ symbols, subject to the restriction that each column contains three distinct symbols. Then clearly, the number of three rowed reduced Latin rectangles on $N$ columns is $\left[t^{N} / N !\right] L(t, 1,1,0,0,0)$, by letting $a$ be row 2 and $b^{-1}$ be row 3 in the summation defining $L$, and by noting that $l(b)=l\left(b^{-1}\right)$ and $g(b)=g\left(b^{-1}\right)$. Goulden and Jackson $[4]$ have shown that

$$
L(t, 1,1,0,0,0)=e^{2 t} \sum_{N \geq 0} \frac{N ! t^{N}}{(1+t)^{3(N+1)}} .
$$

Gessel [3] has shown by a combinatorial argument that

$$
L(t, u, v, 0,0,0)=e^{2 u v t} \sum_{N \geq 0} \frac{t^{N}}{N !} \frac{(u)^{(N)}(v)^{(N)}}{(1+u t)^{v+N}(1+v t)^{u+N}(1+t)^{u v+N}}
$$

is the generating function for three rowed reduced Latin rectangles with $u$ and $v$ marking cycles in rows 2 and 3 respectively, and, more generally, that

$$
L(t, u, v, p, 0,0)=e^{u v t(2-p)} \sum_{N \geq 0} \frac{t^{N}}{N !} \frac{(u)^{(N)}(v)^{(N)}}{(1+u t)^{v+N}(1+v t)^{u+N}(1+(1-p) t)^{u v+N}}
$$

In this section we derive an explicit expression for $L(t, u, v, p, q, r)$, which is denoted hereafter by $L$, as a consequence of Theorem 2.1, by first expressing it as a character sum.

Proposition 3.1. Let $B(\theta, u, q)=(1 / N !) \sum_{\alpha \in \mathbf{S}_{N}} \chi_{\alpha}^{\tilde{\theta}} u^{l(\alpha)} q^{g(\alpha)}$ where $N=$ $|\theta|$. Then

$$
L(t, u, v, p, q, r)=\sum_{N \geq 0} t^{N} \sum_{\theta \vdash N} \frac{N !}{f^{\theta}} B(\theta, p) B(\theta, u, q) B(\theta, v, r)
$$

where $B(\theta, p)$ denotes $B(\theta, 1, p)$.

Proof. Direct from Propositions 2.1, 2.2 and Lemma 2.4 of [5], and since $f^{\theta}=f^{\tilde{\theta}}$.

To evaluate $B(\theta, p)$, it is convenient to use the mapping $\Phi$ such that $\Phi(x)_{k}=$ $(1-p)^{k}$, extended linearly to the appropriate ring. A similar linear functional has been considered by Rota [7].

Proposition 3.2. Let $\theta \vdash N$ and $l(\theta) \leq n$. Then

$$
\frac{N !}{f^{\theta}} B(\theta, p)=(-1)^{N}(1-p)^{N-n} \Phi \prod_{k=1}^{n}\left\{x-\theta_{k}-n+k\right\} .
$$

ProOF. From [6, pp. 25, 62],

$$
B(\theta, p)=s_{\tilde{\theta}}\left\langle p_{1} \mapsto p, p_{i} \mapsto 1, i \geq 2\right\rangle=\left\|\left[e_{\theta_{i}-i+j}\left\langle p_{1} \mapsto p, p_{i} \mapsto 1, i \geq 2\right\rangle\right]\right\|_{n \times n} .
$$


But $\sum_{k \geq 0} e_{k} t^{k}=\exp \sum_{k \geq 1}(-1)^{k-1} p_{k} t^{k} / k$ from [6, p. 17], so making the above substitutions gives $\sum_{k \geq 0} e_{k} t^{k}=(1+t) e^{-(1-p) t}$. Thus

$$
B(\theta, p)=(p-1)^{N}\left\|\left(\theta_{i}-i+j\right) !^{-1}+(p-1)^{-1}\left(\theta_{i}-i+j-1\right) !^{-1}\right\|_{n \times n},
$$

with the convention that $k !^{-1}=0$ for $k<0$.

To evaluate this determinant, we first border it with a first row $(1,0, \ldots, 0)$ and a first column $\left(1,\left(\theta_{1}-1\right) !^{-1}, \ldots,\left(\theta_{n}-n\right) !^{-1}\right)^{T}$. Indexing the rows and columns of the bordered matrix from 0 , replace column $j$ by column $j$ plus $(1-p)^{-1}$ times column $j-1$, first for $j=1$, then for $j=2, \ldots$, and finally for $j=n$. These operations leave the determinant unchanged. Next, multiply in the $k$ th row by $\left(\theta_{k}+n-k\right)$ !, for $k=1, \ldots, n$ and divide the determinant by $\prod_{k=1}^{n}\left(\theta_{k}+n-k\right) !$ to get

$$
B(\theta, p)=\frac{(-1)^{N}(1-p)^{N-n}}{\prod_{k=1}^{n}\left(\theta_{k}+n-k\right) !} \cdot\left\|\frac{\left[(1-p)^{n-j}\right]_{j=0, \ldots, n}}{\left[\left(\theta_{i}+n-i\right)_{n-j}\right]_{i=1, \ldots, n}}\right\| .
$$

The first row can be written in the same form as the other rows since $(1-p)^{n-j}=$ $\Phi\left(\theta_{0}+n\right)_{n-j}$ where $\theta_{0}+n=x$, so by the linearity of $\Phi$ and the multilinearity of the determinant function in its rows

$$
B(\theta, p)=\frac{(-1)^{N}(1-p)^{N-n}}{\prod_{k=1}^{n}\left(\theta_{k}+n-k\right) !} \Phi\left\|\left[\left(\theta_{i}+n-i\right)_{n-j}\right]_{i, j=0, \ldots, n}\right\| .
$$

To complete the evaluation of $B(\theta, p)$, let $\theta_{i}+n-i=\mu_{i}$ and note that $\left(\mu_{i}\right)_{j}$ is a monic polynomial of degree $j$ in $\mu_{i}$. Thus $\left(\mu_{i}\right)_{j}=\sum_{k=0}^{n} c_{k j} \mu_{i}^{k}$ and $C=$ $\left[c_{i j}\right]_{i, j=0, \ldots, n}$ is lower triangular with unit diagonal. Thus the determinant in the expression for $B(\theta, p)$ is equal to

$$
\left\|\sum_{k=0}^{n} c_{n-k n-j} \mu_{i}^{n-k}\right\|=\left\|\left[\mu_{i}^{n-j}\right]_{i, j=0, \ldots, n}\right\| \cdot|C|=\prod_{0 \leq i<j \leq n}\left(\theta_{i}-i-\theta_{j}+j\right)
$$

since the first matrix is Vandermonde, and $|C|=1$. The result follows from the degree formula $\left[6\right.$, p. 64] for $f^{\theta}$.

From the Jacobi-Trudi identity [6, p. 25], another expression for $B(\theta, p)$ as a determinant is

$$
\left\|h_{\tilde{\theta}_{i}-i+j}\left\langle p_{1} \mapsto p, p_{i} \mapsto 1, i \geq 2\right\rangle\right\|_{m \times m}
$$

where $m \geq \theta_{1}$. But $\sum_{k \geq 0} h_{k} t^{k}=\exp \sum_{k \geq 1} p_{k} t^{k} / k$, from [6, p. 25], so making the above power sum substitutions gives $\sum_{k \geq 0} h_{k} t^{k}=(1-t)^{-1} e^{-(1-p) t}$. Thus

$$
B(\theta, p)=\left\|\frac{d_{\tilde{\theta}_{i}-i+j}(p)}{\left(\tilde{\theta}_{i}-i+j\right) !}\right\|_{m \times m}
$$

where $d_{k}(p)=k ! \sum_{i=0}^{k}(p-1)^{i} / i$ !, and $d_{k}(0)$ is therefore the $k$ th derangement number (the number of elements in $\mathbf{S}_{N}$ with no fixed points).

THEOREM 3.3 .

$$
\begin{aligned}
L= & e^{u v t(2-p-q-r+p q r)} \\
& \cdot \sum_{j \geq 0} \frac{t^{j}}{j !} \frac{(u)^{(j)}(v)^{(j)}}{(1+u(1-q) t)^{j+v}(1+v(1-r) t)^{j+u}(1+(1-p) t)^{j+u v}} .
\end{aligned}
$$


PROOF. Let $\hat{p}=1-p, \hat{q}=1-q$, and $\hat{r}=1-r$. From [6, p. 62] we have $B(\theta, u, q)=s_{\theta}\left\langle p_{1} \mapsto q u ; p_{i} \mapsto(-1)^{i-1} u, i \geq 2\right\rangle$. Thus, from Propositions 3.1 and 3.2 ,

$$
L=\Delta \Omega \lim _{n \rightarrow \infty} \hat{p}^{-n} \Phi \sum_{\theta} \prod_{i=1}^{n}\left(x-\theta_{i}-n+i\right) s_{\theta}\left(y_{1}, \ldots, y_{n}\right) s_{\theta}(w)
$$

where $\Delta, \Omega$ are commuting ring homomorphisms defined by their actions

$$
\begin{aligned}
& \Delta: p_{1}(y) \mapsto t \hat{p} v r, \quad p_{i}(y) \mapsto-(-t \hat{p})^{i} v, \quad i \geq 2, \\
& \Omega: p_{1}(w) \mapsto-q u, \quad p_{i}(w) \mapsto-u, \quad i \geq 2,
\end{aligned}
$$

on the power sums, which are algebraically independent $[6$, p. 16]. Now apply Theorem 2.1, to get

$$
\begin{aligned}
L= & \Delta \Omega \lim _{n \rightarrow \infty} \hat{p}^{-n} \Phi \prod_{j=1}^{n} \prod_{k=1}^{\infty}\left(1-y_{j} w_{k}\right)^{-1} \\
& \cdot\left[t_{1} \cdots t_{n}\right]\left(1+t_{1}+\cdots+t_{n}\right)^{x} \prod_{k=1}^{\infty}\left(1-\sum_{j=1}^{n} \frac{t_{j} y_{j} w_{k}}{1-y_{j} w_{k}}\right) .
\end{aligned}
$$

Now let $t_{1}^{2}=\cdots=t_{n}^{2}=0$, which leaves the action of $\left[t_{1} \cdots t_{n}\right]$ unchanged, to obtain

$$
\hat{p}^{-n} \Phi\left(1+t_{1}+\cdots+t_{n}\right)^{x}=\hat{p}^{-n} \exp \left\{\hat{p}\left(t_{1}+\cdots+t_{n}\right)\right\}=\prod_{j=1}^{n}\left(\hat{p}^{-1}+t_{j}\right) .
$$

Applying exp log gives

$$
\begin{aligned}
\Omega \prod_{k=1}^{\infty}\left(1-\sum_{j=1}^{n} \frac{t_{j} y_{j} w^{k}}{1-y_{j} w_{k}}\right) & =\Omega \exp \left(\sum_{i \geq 1} p_{i}(w)\left[\alpha^{i}\right] \log \left(1-\sum_{j=1}^{n} \frac{t_{j} y_{j} \alpha}{1-y_{j} \alpha}\right)\right) \\
& =\exp \left\{-u \log \left(1-\sum_{j=1}^{n} \frac{t_{j} y_{j}}{1-y_{j}}\right)-u \hat{q} \sum_{j=1}^{n} t_{j} y_{j}\right\} \\
& =\left(1-\sum_{j=1}^{n} \frac{t_{j} y_{j}}{1-y_{j}}\right)^{-u} e^{-u \hat{q} \sum_{j=1}^{n} t_{j} y_{j}} \\
& =\left(1-\sum_{j=1}^{n} \frac{t_{j} y_{j}}{1-y_{j}}\right)^{-u} \prod_{j=1}^{n}\left(1-u \hat{q} y_{j} t_{j}\right)
\end{aligned}
$$

where, in the last equality, we have again used $t_{i}^{2}=0$. Similarly

$$
\begin{aligned}
\Omega \prod_{j=1}^{n} \prod_{k=1}^{\infty}\left(1-y_{j} w_{k}\right)^{-1} & =\Omega \exp \sum_{j=1}^{n} \sum_{i \geq 1} \frac{1}{i} y_{j}^{i} p_{i}(w) \\
& =\Omega \exp \left\{-u \sum_{j=1}^{n} \log \left(1-y_{j}\right)^{-1}+u \hat{q} \sum_{j=1}^{n} y_{j}\right\} \\
& =\prod_{j=1}^{n}\left(1-y_{j}\right)^{u} e^{u \hat{q} \sum_{j=1}^{n} y_{j}} .
\end{aligned}
$$


Combining these results yields

$$
\begin{aligned}
L= & \Delta \lim _{n \rightarrow \infty} \prod_{j=1}^{n}\left(1-y_{j}\right)^{u} e^{u \hat{q} \sum_{j=1}^{n} y_{j}} \\
& \cdot\left[t_{1} \cdots t_{n}\right]\left(1-\sum_{j=1}^{n} \frac{t_{j} y_{j}}{1-y_{j}}\right)^{-u} \prod_{j=1}^{n}\left(\hat{p}^{-1}+t_{j}\right)\left(1-u \hat{q} y_{j} t_{j}\right) .
\end{aligned}
$$

To assist in the application of the operator $\left[t_{1} \cdots t_{n}\right]$ let $\Psi\left(z^{i}\right)=(u)^{(i)}, i \geq 0$, extended linearly. Then

$$
\begin{aligned}
\left(1-\sum_{j=1}^{n} \frac{t_{j} y_{j}}{1-y_{j}}\right)^{-u} & =\sum_{i \geq 0} \frac{1}{i !}(u)^{(i)}\left(\sum_{j=1}^{n} \frac{t_{j} y_{j}}{1-y_{j}}\right)^{i}=\Psi \sum_{i \geq 0} \frac{z^{i}}{i !}\left(\sum_{j=1}^{n} \frac{t_{j} y_{j}}{1-y_{j}}\right)^{i} \\
& =\Psi \exp \left(z \sum_{j=1}^{n} \frac{t_{j} y_{j}}{1-y_{j}}\right)=\Psi \prod_{j=1}^{n}\left(1+\frac{t_{j} y_{j} z}{1-y_{j}}\right),
\end{aligned}
$$

so, since $\Psi$ and $\Delta$ commute

$$
\begin{aligned}
L= & \Psi \Delta \lim _{n \rightarrow \infty} e^{u \hat{q} \sum_{j=1}^{n} y_{j}} \prod_{j=1}^{n}\left(1-y_{j}\right)^{u} \\
& \cdot\left[t_{1} \cdots t_{n}\right] \prod_{j=1}^{n}\left(\hat{p}^{-1}+t_{j}\right)\left(1-u \hat{q} y_{j} t_{j}\right)\left(1+\frac{t_{j} y_{j} z}{1-y_{j}}\right) \\
= & \Psi \Delta \lim _{n \rightarrow \infty} e^{u \hat{q} \sum_{j=1}^{n} y_{j}} \prod_{j=1}^{n}\left(1-y_{j}\right)^{u}\left(1-u \frac{\hat{q}}{\hat{p}} y_{j}+\frac{y_{j} z}{\hat{p}\left(1-y_{j}\right)}\right) \\
= & \Psi \Delta e^{u \hat{q} p_{1}(y)} \prod_{j \geq 1}\left(1-y_{j}\right)^{u}\left(1-u \frac{\hat{q}}{\hat{p}} y_{j}+\frac{y_{j} z}{\hat{p}\left(1-y_{j}\right)}\right) \\
= & \Psi \Delta \exp \left(u \hat{q} p_{1}(y)+\sum_{i \geq 1} p_{i}(y)\left[\alpha^{i}\right] \log \left\{(1-\alpha)^{u}\left(1-u \frac{\hat{q}}{\hat{p}} \alpha+\frac{\alpha z}{\hat{p}(1-\alpha)}\right)\right\}\right) \\
= & \Psi \exp (u \hat{q} t \hat{p} v r \\
= & \left.\left.\Psi(1+\hat{p} t)^{-u v}\left(1+u \hat{q} t-\frac{t z}{1+\hat{p} t}\right)^{-v} \exp (1+\hat{p} t)^{u}\left(1+u \hat{q} t-\frac{t z}{1+\hat{p} t}\right)\right\}+v \hat{r}\{u \hat{p} t+u \hat{q} t-t z\}\right) \\
= & \frac{e^{u v t(2-p-q-r+p q r)}}{(1+\hat{p} t)^{u v}(1+u \hat{q} t)^{v}} \Psi\left(1-\frac{z t}{(1+\hat{p} t)(1+u \hat{q} t)}\right)^{-v} e^{-v t \hat{r} z} \\
= & e^{u v t(2-p-q-r+p q r)} \sum_{j \geq 0} \frac{(v)^{(j)}}{j !} \frac{t^{j}}{(1+\hat{p} t)^{u v+j}(1+u \hat{q} t)^{v+j}} \Psi z^{j} e^{-v t \hat{r} z} .
\end{aligned}
$$


But

$$
\begin{aligned}
\Psi\left\{z^{j} e^{\alpha z}\right\} & =\sum_{i \geq 0} \frac{\alpha^{i}}{i !} \Psi z^{i+j}=\sum_{i \geq 0} \frac{\alpha^{i}}{i !}(u)^{(i+j)} \\
& =(u)^{(j)} \sum_{i \geq 0} \frac{\alpha^{i}}{i !}(u+j)^{(i)}=(u)^{(j)}(1-\alpha)^{-u-j}
\end{aligned}
$$

and the result follows with $\alpha=-\hat{r} v t$.

With $p=q=r=0$, Theorem 3.3 specializes to Gessel's result [3]. With $p=q=r=1$ it specializes to the generating function $\sum_{j \geq 0} t^{j}(u)^{(j)}(v)^{(j)} / j$ ! for pairs of permutations with a specified number of cycles in each permutation.

Further refinements of the counting problem can be obtained by following the method of proof of Theorem 3.3. Instead of marking the cycles in the second permutation as we have, consider marking $i$-cycles by an indeterminate $v_{i}$, for $i=1,2, \ldots$. Then the action of the homomorphism $\Delta$ defined in Theorem 3.3 is modified to $p_{i}(w) \mapsto-(-t \hat{p})^{i} v_{i}$, for $i \geq 1$. This can be carried out explicitly in terms of the roots $\beta, \gamma$ of

$$
x^{2}-\left(1+u \frac{\hat{q}}{\hat{p}}-\frac{z}{\hat{p}}\right) x+u \frac{\hat{q}}{\hat{p}}=0
$$

as a quadratic in $x$. Then the required generating function is

$$
\Psi \exp \left\{u \hat{q} t \hat{p} v_{1}-\sum_{i \geq 1}(-t \hat{p})^{i} \frac{v_{i}}{i}\left(1-u-\beta^{i}-\gamma^{i}\right)\right\} .
$$

The interested reader may wish to complete this argument.

4. Equivalent forms and some specializations. A generalization of the summation theorem

$$
\sum_{\theta} s_{\theta}\left(y_{1}, \ldots, y_{n}\right) s_{\tilde{\theta}}\left(w_{1}, \ldots, w_{m}\right)=\prod_{j=1}^{n} \prod_{k=1}^{m}\left(1+y_{j} w_{k}\right)
$$

also due to Cauchy (see [6, p. 35] for a proof), can be deduced from Theorem 2.1.

COROLlaRY 4.1. For nonnegative integers $n$ and $m$,

$$
\begin{aligned}
& \sum_{\theta} \prod_{i=1}^{n}\left(x-\theta_{i}-n+i\right) s_{\theta}\left(y_{1}, \ldots, y_{n}\right) s_{\tilde{\theta}}\left(w_{1}, \ldots, w_{m}\right) \\
& \quad=\prod_{j=1}^{n} \prod_{k=1}^{m}\left(1+y_{j} w_{k}\right)\left[t_{1} \cdots t_{n}\right]\left(1+t_{1}+\cdots+t_{n}\right)^{x} \prod_{k=1}^{m}\left(1+\sum_{j=1}^{n} \frac{t_{j} y_{j} w_{k}}{1+y_{j} w_{k}}\right)^{-1} .
\end{aligned}
$$

PROOF. Consider the mapping $\omega$ defined on the ring of symmetric functions in $w_{1}, w_{2}, \ldots$ by $\omega\left(e_{k}\right)=h_{k}$ (the $e_{r}$ are algebraically independent $\left.[6, p .14]\right)$. Then $\left[6\right.$, p. 14] $\omega$ is an involution, and $\omega s_{\theta}=s_{\tilde{\theta}}$ and $\omega p_{i}=(-1)^{i-1} p_{i}$ for $i \geq 1$. Letting 
$m \rightarrow \infty$ in Theorem 2.1 and applying $\omega$ to both sides of this theorem gives

$$
\begin{aligned}
\sum_{\theta} \prod_{i=1}^{n}\left(x-\theta_{i}-n+i\right) s_{\theta}\left(y_{1}, \ldots, y_{n}\right) s_{\tilde{\theta}}(w) \\
=\omega \exp \left\{\sum_{i \geq 1} \frac{1}{i} p_{i}\left(y_{1}, \ldots, y_{n}\right) p_{i}(w)\right\}\left[t_{1} \cdots t_{n}\right]\left(1+t_{1}+\cdots+t_{n}\right)^{x} \\
\quad \cdot \exp \left\{\sum_{i \geq 1} p_{i}(w)\left[\alpha^{i}\right] \log \left(1-\sum_{j=1}^{n} \frac{t_{j} y_{j} \alpha}{1-y_{j} \alpha}\right)\right\}
\end{aligned}
$$

in which exp log has been applied to the right-hand side to obtain an expansion of the products. The result is obtained now by applying $\omega$ to $p_{i}(w), i \geq 1$, and then setting $w_{i}=0$ for $i \geq m+1$.

For any partition $\theta$, with $l(\theta) \leq n$, the power series $S_{\theta}(w ; z)$, which arise in connexion with the Hall-Littlewoood polynomials [6, p. 104], are defined by

$$
S_{\theta}(w ; z)=\left\|q_{\theta_{i}-i+j}(w ; z)\right\|_{n \times n}
$$

where

$$
\sum_{k \geq 0} q_{k}(w ; z) t^{k}=\prod_{i \geq 1} \frac{1-t w_{i} z}{1-t w_{i}} .
$$

Then, from [6, pp. 106, 117],

$$
\sum_{\theta} s_{\theta}\left(y_{1}, \ldots, y_{n}\right) S_{\theta}\left(w_{1}, \ldots, w_{m} ; z\right)=\prod_{j=1}^{n} \prod_{k=1}^{m} \frac{1-y_{j} w_{k} z}{1-y_{j} w_{k}} .
$$

A generalization of this summation theorem is contained in the following result, which is also deduced from Theorem 2.1.

COROLLARY 4.2. For nonnegative integers $n$ and $m$,

$$
\begin{aligned}
\sum_{\theta} \prod_{i=1}^{n}\left(x-\theta_{i}-n+i\right) s_{\theta}\left(y_{1}, \ldots, y_{n}\right) S_{\theta}\left(w_{1}, \ldots, w_{m} ; z\right) \\
=\prod_{j=1}^{n} \prod_{k=1}^{m} \frac{1-z y_{j} w_{k}}{1-y_{j} w_{k}}\left[t_{1} \cdots t_{n}\right]\left(1+t_{1}+\cdots+t_{n}\right)^{x} \\
\quad \cdot \prod_{k=1}^{m}\left(1-\sum_{j=1}^{n} \frac{t_{j} y_{j} w_{k}}{1-y_{j} w_{k}}\right)\left(1-\sum_{j=1}^{n} \frac{t_{j} y_{j} w_{k} z}{1-y_{j} w_{k} z}\right)^{-1} .
\end{aligned}
$$

PROOF. Follow the proof of Corollary 4.1, but use the homomorphism which maps $s_{\theta}$ onto $S_{\theta}$. This homomorphism also maps $p_{i}$ onto $\left(1-z^{i}\right) p_{i}$, and the result follows.

Theorem 2.1 can also be used to derive a result for applying the formal partial differential operator $\prod_{i=1}^{n}\left(x-y_{i} \partial / \partial y_{i}\right)$ to a special type of anti-symmetric function in $y_{1}, \ldots, y_{n}$. This operator was denoted by $Y_{0}$ in the proof of Theorem 2.1. For the following result, let $A * B=\sum_{i \geq 0} a_{i} b_{i}$ where $A=\sum_{i \geq 0} a_{i} \alpha^{i}$ and $B=\sum_{i \geq 0} b_{i} \alpha^{i}$. 
THEOREM 4.3. Let $F(y)$ be a formal power series in y with constant term equal to 1 . Then

$$
\begin{aligned}
\prod_{i=1}^{n}\left(x-y_{i} \frac{\partial}{\partial y_{i}}\right) a_{\delta} F\left(y_{1}\right) \cdots F\left(y_{n}\right) \\
=a_{\delta} F\left(y_{1}\right) \cdots F\left(y_{n}\right)\left[t_{1} \cdots t_{n}\right]\left(1+t_{1}+\cdots+t_{n}\right)^{x} \\
\quad \cdot \exp \left\{\left(\alpha \frac{\partial}{\partial \alpha} \log F(\alpha)\right) * \log \left(1-\sum_{j=1}^{n} \frac{t_{j} y_{j} \alpha}{1-y_{j} \alpha}\right)\right\} .
\end{aligned}
$$

PROOF. Equation (1) in the proof of Theorem 2.1 is equivalent to Theorem 2.1 itself. Let $m \rightarrow \infty$ and apply exp log to the products on both sides of this equation to obtain

$$
\begin{aligned}
\prod_{i=1}^{n}\left(x-y_{i} \frac{\partial}{\partial y_{i}}\right) a_{\delta} \exp \left\{\sum_{i \geq 1} \frac{1}{i} p_{i}\left(y_{1}, \ldots, y_{n}\right) p_{i}(w)\right\} \\
=a_{\delta} \exp \left\{\sum_{i \geq 1} \frac{1}{i} p_{i}\left(y_{1}, \ldots, y_{n}\right) p_{i}(w)\right\}\left[t_{1} \cdots t_{n}\right]\left(1+t_{1}+\cdots+t_{n}\right)^{x} \\
\quad \cdot \exp \left\{\sum_{i \geq 1} p_{i}(w)\left[\alpha^{i}\right] \log \left(1-\sum_{j=1}^{n} \frac{t_{j} y_{j} \alpha}{1-y_{j} \alpha}\right)\right\} .
\end{aligned}
$$

But the power sum symmetric functions are algebraically independent (see $[6, \mathrm{p}$. 16]) so $p_{i}(w)$ in the above equality can be replaced by the indeterminate $c_{i}$, for $i \geq 1$. If we carry out this replacement, we obtain

$$
\exp \sum_{i \geq 1} \frac{1}{i} p_{i}\left(y_{1}, \ldots, y_{n}\right) p_{i}(w)=\exp \sum_{i \geq 1} \frac{c_{i}}{i} p_{i}\left(y_{1}, \ldots, y_{n}\right)=\prod_{j=1}^{n} \exp \left(\sum_{i \geq 1} \frac{c_{i}}{i} y_{i}^{j}\right) .
$$

Now let $F(y)=\exp \left(\sum_{i \geq 1} c_{i} y^{i} / i\right)$ and the result follows, since

$$
\sum_{i \geq 1} c_{i} \alpha^{i}=\alpha \frac{\partial}{\partial \alpha} \log F(\alpha) .
$$

Many specializations of Theorem 4.3 are possible. We give one such result as an example.

COROLLARY 4.4. For nonnegative integers $n, k$,

$$
\begin{aligned}
\prod_{i=1}^{n} & \left(x-y_{i} \frac{\partial}{\partial y_{i}}\right) a_{\delta}\left(y_{1}+\cdots+y_{n}\right)^{k} \\
& =a_{\delta} \sum_{j=0}^{k}(-1)^{j}(k)_{j}(x)_{n-j}\left(y_{1}+\cdots+y_{n}\right)^{k-j} e_{j}\left(y_{1}, \ldots, y_{n}\right) .
\end{aligned}
$$


PROOF. Let $F(y)=e^{z y}$ in Theorem 4.3 to obtain

$$
\begin{aligned}
\prod_{i=1}^{n}\left(x-y_{i} \frac{\partial}{\partial y_{i}}\right) a_{\delta} e^{z\left(y_{1}+\cdots+y_{n}\right)}= & a_{\delta} e^{z\left(y_{1}+\cdots+y_{n}\right)}\left[t_{1} \cdots t_{n}\right]\left(1+t_{1}+\cdots+t_{n}\right)^{x} \\
& \cdot \exp \left\{(\alpha z) * \log \left(1-\sum_{j=1}^{n} \frac{t_{j} y_{j} \alpha}{1-y_{j} \alpha}\right)\right\} .
\end{aligned}
$$

But

$$
(\alpha z) * \log \left(1-\sum_{j=1}^{n} \frac{t_{j} y_{j} \alpha}{1-y_{j} \alpha}\right)=-z \sum_{j=1}^{n} t_{j} y_{j},
$$

and the result follows by equating coefficients of $z^{k} / k$ !

Theorem 4.3, as stated, allows us to evaluate the given left-hand side in the form $\left[t_{1} \cdots t_{n}\right] G$ for some power series $G$. The proof is indirect, relying on induction and interpolation. There are, however, a number of ways of writing the left-hand side directly in this form, but for different choices of $G$. One such way is given in the next result.

THEOREM 4.5. Let $F(y)$ be a power series in $y$ with constant term 1. Then for any nonnegative integer $n$

$$
\begin{aligned}
\prod_{i=1}^{n}( & \left.x-y_{i} \frac{\partial}{\partial y_{i}}\right) a_{\delta}\left(y_{1}, \ldots, y_{n}\right) F\left(y_{1}\right) \cdots F\left(y_{n}\right) \\
= & {\left[t_{1} \cdots t_{n}\right] e^{x\left(t_{1}+\cdots+t_{n}\right)} a_{\delta}\left(y_{1}-t_{1} y_{1}, \ldots, y_{n}-t_{n} y_{n}\right) } \\
& \cdot F\left(y_{1}-t_{1} y_{1}\right) \cdots F\left(y_{n}-t_{n} y_{n}\right) .
\end{aligned}
$$

PROOF. Clearly, the action of the differential operator on the left-hand side is the same as the action of the operator

$$
\begin{array}{r}
{\left[t_{1} \ldots t_{n}\right] \prod_{i=1}^{n} \sum_{k_{i} \geq 0} \frac{1}{k_{i} !}\left\{t_{i}\left(x-y_{i} \frac{\partial}{\partial y_{i}}\right)\right\}^{k_{i}}} \\
=\left[t_{1} \cdots t_{n}\right] \prod_{i=1}^{n} \exp \left\{t_{i}\left(x-y_{i} \frac{\partial}{\partial y_{i}}\right)\right\} \\
=\left[t_{1} \cdots t_{n}\right] e^{x\left(t_{1}+\cdots+t_{n}\right)} \prod_{i=1}^{n} e^{-t_{i} y_{i} \partial / \partial y_{i}}
\end{array}
$$

since $\partial / \partial y_{i}$ and $\partial / \partial y_{j}$ commute for all $i$ and $j$. But, for any power series $G(y)$

$$
e^{t y \partial / \partial y} G(y)=G\left(e^{t} y\right) \text {. }
$$

Thus the left-hand side of the result is equal to

$$
\left[t_{1} \cdots t_{n}\right] e^{x\left(t_{1}+\cdots+t_{n}\right)} a_{\delta}\left(e^{-t_{1}} y_{1}, \ldots, e^{-t_{n}} y_{n}\right) F\left(e^{-t_{1}} y_{1}, \ldots, e^{-t_{n}} y_{n}\right)
$$

The result follows by replacing $e^{-t_{i}}$ with $1-t_{i}$ for $i=1, \ldots, n$, since this leaves the action of $\left[t_{1} \cdots t_{n}\right]$ unchanged.

Combining Theorems 4.3 and 4.5 gives a pair of different power series in which the coefficient of $t_{1} \cdots t_{n}$ is identical. As an example, we now give one of the many 
results which can be obtained as special cases of the identity. The result involves the Stirling numbers of the first kind, defined by $s(n, k)=\left[x^{k}\right](x)_{n}$ (see [6, p. 22]). This is equal to $(-1)^{n-k}$ times the number of permutations on $n$ symbols with exactly $k$ cycles.

COROLlary 4.6. For all $0 \leq k \leq n$,

$$
s(n, k)=\left[t_{1} \cdots t_{n}\right] e_{k}\left(t_{1}, \ldots, t_{n}\right) \prod_{1 \leq i<j \leq n}\left(1-\frac{t_{i} y_{i}-t_{j} y_{j}}{y_{i}-y_{j}}\right) .
$$

PROOF. Equate the right-hand sides of Theorem 4.5 and 4.3 , with $F(y) \equiv 1$, to obtain

$$
\begin{aligned}
& a_{\delta}\left(y_{1}, \ldots, y_{n}\right)\left[t_{1} \cdots t_{n}\right]\left(1+t_{1}+\cdots+t_{n}\right)^{x} \\
& \quad=\left[t_{1} \cdots t_{n}\right] \exp ^{x\left(t_{1}+\cdots+t_{n}\right)} a_{\delta}\left(y_{1}-t_{1} y_{1}, \ldots, y_{n}-t_{n} y_{n}\right) .
\end{aligned}
$$

The result follows by equating coefficients of $x^{k}$, and by dividing on both sides by $a_{\delta}\left(y_{1}, \ldots, y_{n}\right)$.

5. Some related determinants. Closely related to Cauchy's theorem from Schur functions is Cauchy's determinant formula:

$$
\left\|\frac{1}{1-y_{i} w_{j}}\right\|_{n \times n}=\frac{a_{\delta}\left(y_{1}, \ldots, y_{n}\right) a_{\delta}\left(w_{1}, \ldots, w_{n}\right)}{\prod_{i=1}^{n} \prod_{j=1}^{n}\left(1-y_{i} w_{j}\right)}
$$

(see [6, p. 38] for a proof). As a corollary of Theorem 2.1, we obtain Borchardt's [10] striking result involving Cauchy's determinant. Throughout this section, all matrices are $n \times n$ unless otherwise stated. The permanent of the matrix whose $i j$-element is $m_{i j}$ is denoted by $\left\|m_{i j}\right\|^{+}$.

COROLlaRY 5.1 .

$$
\left\|\frac{1}{\left(1-y_{i} w_{j}\right)^{2}}\right\|=\left\|\frac{1}{1-y_{i} w_{j}}\right\| \cdot\left\|\frac{1}{1-y_{i} w_{j}}\right\|^{+} .
$$

PROOF. Equation (1) in the proof of Theorem 2.1 is equivalent to Theorem 2.1. In this equation, let $x=0$ and $m=n$, multiply on both sides by $a_{\delta}$ and then apply Cauchy's determinant formula to obtain

$$
\prod_{i=1}^{n}\left(-y_{i} \frac{\partial}{\partial y_{i}}\right)\left\|\frac{1}{1-y_{i} w_{j}}\right\|=\left\|\frac{1}{1-y_{i} w_{j}}\right\|\left[t_{1} \cdots t_{n}\right] \prod_{j=1}^{n}\left(1-\sum_{i=1}^{n} \frac{t_{i} y_{i} w_{j}}{1-y_{i} w_{j}}\right) \text {. }
$$

But

and

$$
\begin{aligned}
\prod_{i=1}^{n}\left(-y_{i} \frac{\partial}{\partial y_{i}}\right)\left\|\frac{1}{1-y_{i} w_{j}}\right\| & =\left\|-y_{i} \frac{\partial}{\partial y_{i}}\left(\frac{1}{1-y_{i} w_{j}}\right)\right\| \\
& =\left\|\frac{-y_{i} w_{j}}{\left(1-y_{i} w_{j}\right)^{2}}\right\|
\end{aligned}
$$

$$
\left[t_{1} \cdots t_{n}\right] \prod_{j=1}^{n}\left(1-\sum_{i=1}^{n} \frac{t_{i} y_{i} w_{j}}{1-y_{i} w_{j}}\right)=\left\|\frac{-y_{i} w_{j}}{1-y_{i} w_{j}}\right\|^{+} .
$$

Combine these results, and divide both sides by $(-1)^{n} y_{1} \cdots y_{n} w_{1} \cdots w_{n}$ to obtain the result.

The left-hand side of Theorem 4.3 can be written as a determinant also, and this is given in the next result. 
THEOREM 5.2. Let $F(y)$ be a power series with constant term 1. Then for $n$, a nonnegative integer,

$$
\begin{aligned}
& \prod_{i=1}^{n}\left(x-y_{i} \frac{\partial}{\partial y_{i}}\right) a_{\delta} F\left(y_{1}\right) \cdots F\left(y_{n}\right) \\
& \quad=F\left(y_{1}\right) \cdots F\left(y_{n}\right)\left\|\left(x-n+j-y_{i} \frac{\partial}{\partial y_{i}}\left\{\log F\left(y_{i}\right)\right\}\right) y_{i}^{n-j}\right\| .
\end{aligned}
$$

Proof. We have

$$
a_{\delta} F\left(y_{1}\right) \cdots F\left(y_{n}\right)=F\left(y_{1}\right) \cdots F\left(y_{n}\right)\left\|y_{i}^{n-j}\right\|=\left\|y_{i}^{n-j} F\left(y_{i}\right)\right\|,
$$

so

$$
\prod_{i=1}^{n}\left(x-y_{i} \frac{\partial}{\partial y_{i}}\right) a_{\delta} F\left(y_{1}\right) \cdots F\left(y_{n}\right)=\left\|\left(x-y_{i} \frac{\partial}{\partial y_{i}}\right) y_{i}^{n-j} F\left(y_{i}\right)\right\|
$$

and the result follows immediately.

The authors have been unable to exploit Theorem 5.2 to obtain a direct proof of Theorem 4.3. However, Theorem 5.2 does yield a linear recurrence for the left-hand side of Theorem 4.3. This is stated in the next result, and we give it since it may suggest a more direct proof.

COROLlaRY 5.3. Let $F(y)$ be a power series with constant term 1. Let

$$
H_{n}(y, x)=\prod_{i=1}^{n}\left(x-y_{i} \frac{\partial}{\partial y_{i}}\right) a_{\delta} F\left(y_{1}\right) \cdots F\left(y_{n}\right) .
$$

Then

$$
H_{n}(y, x)=y_{1} \cdots y_{n} \sum_{i=1}^{n}(-1)^{n-i} y_{i}^{-1} F\left(y_{i}\right)\left(x-y_{i} \frac{\partial}{\partial y_{i}} \log F\left(y_{i}\right)\right) H_{n-1}\left(\frac{y}{y_{i}}, x-1\right)
$$

where $y / y_{i}=\left(y_{1}, \ldots, y_{i-1}, y_{i+1}, \ldots, y_{n}\right)$.

PROOF. The result follows straightforwardly from Theorem 5.2 by a cofactor expansion in the last column of the determinant on the right-hand side.

ACKNOWLEDGMENTS. This work was supported by Grant DMS-8503324 from the National Science Foundation (USA), and by Grants A8907 and A8235 from the Natural Sciences and Engineering Research Council of Canada.

\section{REFERENCES}

1. E. Bannai and T. Ito, Algebraic combinatorics I, Association schemes. Benjamin/Cummings, Menlo Park, Calif., 1984.

2. P. Diaconis, Group theory in statistics [interim title for a book].

3. I. M. Gessel, Counting 3-lined Latin rectangles, Lecture Notes in Math., vol. 1234, Springer, 1985, pp. 106-111.

4. I. P. Goulden and D. M. Jackson, Combinatorial enumeration, Wiley, New York, 1983.

5. D. M. Jackson, Counting cycles in permutations by group characters, with an application to a topological problem, Trans. Amer. Math. Soc. 299 (1987), 785-801.

6. I. G. Macdonald, Symmetric functions and Hall polynomials, Clarendon Press, Oxford, 1979.

7. G.-C. Rota, The number of partitions of a set, Amer. Math. Monthly 71 (1964), 498-504.

8. B. E. Sagan (private communication). 
9. C. Schensted, Longest increasing and decreasing subsequences, Canad. J. Math. 13 (1961), 179-191.

10. C. W. Borchardt, Bestimmung der symmetrischen Verbindungen Vermittelst ihrer erzeugenden Funktion, Montash. Akad. Wiss. Berlin, 1855, pp. 165-171.

Department of Mathematics, Pennsylvania State University, University PARK, PENNSYLVANIA 16802

DEPARTMENT OF COMBINATORICS AND OPTIMIZATION, UNIVERSITY OF WATERLOO, WATERloo, ONtario, CaNAda N2L 3G1 